\title{
Ultra-Narrow SPP Generation from Ag Grating ${ }^{\dagger}$
}

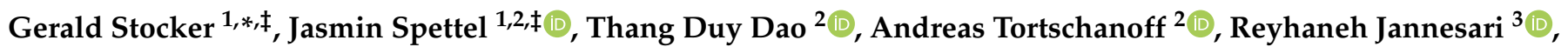 \\ Gerald Pühringer ${ }^{3}$, Parviz Saeidi ${ }^{3}{ }^{\circledR 0}$, Florian Dubois ${ }^{2}$, Clement Fleury ${ }^{2}$, Cristina Consani ${ }^{2}$, Thomas Grille ${ }^{1}$, \\ Elmar Aschauer ${ }^{1}$ and Bernhard Jakoby ${ }^{3}$ (D)
}

1 Infineon Technologies Austria AG, 9500 Villach, Austria; Jasmin.Spettel@silicon-austria.com (J.S.); Thomas.Grille@infineon.com (T.G.); Elmar.Aschauer@infineon.com (E.A.)

2 Silicon Austria Labs GmbH, 9524 Villach, Austria; Thang.Dao@silicon-austria.com (T.D.D.); Andreas.Tortschanoff@silicon-austria.com (A.T.); Florian.Dubois@silicon-austria.com (F.D.); Clement.Fleury@silicon-austria.com (C.F.); Cristina.Consani@silicon-austria.com (C.C.)

3 Institute of Microelectronics and Micro Sensoric, Johannes Kepler University Linz, 4040 Linz, Austria; Reyhaneh.Jannesari@jku.at (R.J.); Gerald.Puehringer@jku.at (G.P.); Parviz.Saeidi@jku.at (P.S.); Bernhard.Jakoby@jku.at (B.J.)

* Correspondence: Gerald.Stocker@infineon.com

$+\quad$ This paper is an extended version of our paper published in Stocker, G.; Spettel, J.; Dao, T.D.; Tortschanoff, A.; Jannesari, R.; Pühringer, G.; Saeidi, P.; Dubois, F.; Fleury, C.; Consani, C.; et al. Plasmonic Silver Grating for Mid-Infrared Sensing. In Proceedings of the 21st International Conference on Solid-State Sensors, Actuators and Microsystems (Transducers), Orlando, FL, USA, 20-24 June 2021; pp. 1072-1075.

$\ddagger \quad$ These authors contributed equally to this work.

Citation: Stocker, G. ; Spettel, J.; Dao, T.D.; Tortschanoff, A.; Jannesari, R.; Pühringer, G.; Saeidi, P.; Dubois, F.; Fleury, C.; Consani, C.; et al. Ultra-Narrow SPP Generation from Ag Grating. Sensors 2021, 21, 6993. https://doi.org/10.3390/s21216993

Academic Editors: Atsushi Motogaito and Elba Mauriz

Received: 11 September 2021

Accepted: 18 October 2021

Published: 21 October 2021

Publisher's Note: MDPI stays neutral with regard to jurisdictional claims in published maps and institutional affiliations.

Copyright: (c) 2021 by the authors. Licensee MDPI, Basel, Switzerland. This article is an open access article distributed under the terms and conditions of the Creative Commons Attribution (CC BY) license (https:// creativecommons.org/licenses/by/ $4.0 /)$.

\begin{abstract}
In this study, we investigate the potential of one-dimensional plasmonic grating structures to serve as a platform for, e.g., sensitive refractive index sensing. This is achieved by comparing numerical simulations to experimental results with respect to the excitation of surface plasmon polaritons (SPPs) in the mid-infrared region. The samples, silver-coated poly-silicon gratings, cover different grating depths in the range of $50 \mathrm{~nm}-375 \mathrm{~nm}$. This variation of the depth, at a fixed grating geometry, allows the active tuning of the bandwidth of the SPP resonance according to the requirements of particular applications. The experimental setup employs a tunable quantum cascade laser (QCL) and allows the retrieval of angle-resolved experimental wavelength spectra to characterize the wavelength and angle dependence of the SPP resonance of the specular reflectance. The experimental results are in good agreement with the simulations. As a tendency, shallower gratings reveal narrower SPP resonances in reflection. In particular, we report on $2.9 \mathrm{~nm}$ full width at half maximum (FWHM) at a wavelength of $4.12 \mu \mathrm{m}$ and a signal attenuation of $21 \%$. According to a numerical investigation with respect to a change of the refractive index of the dielectric above the grating structure, a spectral shift of $4122 \frac{\mathrm{nm}}{\mathrm{RIU}}$ can be expected, which translates to a figure of merit (FOM) of about $1421 \mathrm{RIU}^{-1}$. The fabrication of the suggested structures is performed on eight-inch silicon substrates, entirely accomplished within an industrial fabrication environment using standard microfabrication processes. This in turn represents a decisive step towards plasmonic sensor technologies suitable for semiconductor mass-production.
\end{abstract}

Keywords: surface plasmon polaritons; refractive index sensing; reflection measurement; plasmonic grating

\section{Introduction}

The number and variety of sensor applications has increased dramatically with the development of smart home systems. The sensing of the environment, for example ambient gases, is of growing interest for many applications such as indoor air quality monitoring, gas leakage or fire detection. The miniaturization of existing sensor concepts is one way to meet the demand of integrated sensors. In this context, plasmonics in the mid-infrared (MIR) region and their usage for sensor applications have emerged as a field of interest in 
the recent years [1]. These surface effects allow monitoring different physical or chemical properties at material interfaces in a non-invasive way [2-5] or even several physical properties at once [6,7]. Besides such sensing applications, the list of use-cases in the field of plasmonic research in the mid- and near-infrared region also covers waveguides [8], selective thermal emitters $[9,10]$ and infrared (IR) detectors [11]. For all mentioned applications, the performance directly depends on the physical properties of the used materials and the structural geometry. These properties, together with the fabrication capabilities determine the usability in particular applications.

In this work, we numerically investigate and experimentally demonstrate the excitation of surface plasmon polaritons (SPPs) in the mid-infrared (MIR) region in silver-coated poly-silicon gratings. The study covers a wavelength range of $4.1 \mu \mathrm{m}-4.3 \mu \mathrm{m}$ which is of interest for gas-sensing applications, particularly for $\mathrm{CO}_{2}$ gas sensors. To demonstrate ultra-narrow SPP resonances, we probe plasmonic gratings of four different depths $(50 \mathrm{~nm}$, $150 \mathrm{~nm}, 225 \mathrm{~nm}, 375 \mathrm{~nm}$ ), keeping the grating geometry fixed. A narrowband resonant plasmonic grating with high absorptivity would constitute an ideal platform for e.g., sensitive refractive index sensing $[6,12]$. Plasmonic gratings with a highly confined near-field at the metal-dielectric interface could be applied for surface-enhanced infrared absorption spectroscopy (SEIRA) [13]. In the presented work, the experimental characterization of the samples covers angle-resolved reflection measurements, where employing a tunable quantum cascade laser (QCL) adds another degree of freedom to the experiment. The findings indicate that by increasing the grating depths, the resonant bandwidth (FWHM) tends to increase while the resonant specular reflectance decreases (absorptivity increases). Therefore, the grating with $50 \mathrm{~nm}$ depth revealed the narrowest resonances, for example at $4.12 \mu \mathrm{m}$ wavelength under a $28^{\circ}$ incidence with an FWHM of $1.4 \mathrm{~nm}$ by simulation and $2.9 \mathrm{~nm}$ by experiment. To show the potential of the suggested structure with respect to sensing applications, a numerical investigation concerning sensitive refractive index sensing is presented.

Besides the theoretical and experimental part, we also describe the fabrication of the samples done on eight-inch silicon wafers, entirely performed in a state-of-the-art clean room facility of semiconductor industry. In particular, the feasibility to fabricate plasmonic grating structures for the mid-infrared region within an industrial fabrication environment using standard microfabrication processes represents a decisive step towards plasmonic sensor technologies suitable for semiconductor mass-production.

Preliminary results of this work were presented at the Transducers 2021 Conference and published in its Proceedings [14].This extended paper is intended to provide further and deeper insights to the study.

The remainder of this work is organized as follows. Section 2 describes a short background of the SPP excitation using the grating configuration. This section also discusses the choice of silver as the preferential material for SPPs in the MIR region, as well as the optical measurement setup and the sample fabrication. The angle-measurement results are presented and discussed in Section 3 where we show the strategy how to control the grating depth for the narrowband SPP resonance. Additionally, sensitive refractive index sensing is numerically demonstrated as potential sensing application. Finally, Section 4 summarizes the work.

\section{Materials and Methods}

\subsection{Surface Plasmon Polaritons}

The presented and discussed measurements employ free-beam reflection measurements to probe plasmonic properties of our structures, which are designed to allow the excitation of SPPs at the silver-air interface. SPPs are the collective oscillations of free electrons within a metal-like layer introduced by the interaction with photons at the layer's surface [15]. The resulting wave, propagating along the interface of dielectric (air) and plasmonic layer (silver), is of both photonic and plasmonic nature. The strength of the electric field is exponentially decaying from the interface into both media [16]. To excite 
a SPP by a photon, both need to have the same momentum. At a given frequency, a free space photon has less momentum than a SPP, due to different dispersion relations [15]. The wave number of the SPP, $k_{\mathrm{SPP}}$, is given by

$$
k_{\mathrm{SPP}}=k_{\mathrm{d}} \sqrt{\frac{\epsilon_{\mathrm{d}} \epsilon_{\mathrm{m}}}{\epsilon_{\mathrm{d}}+\epsilon_{\mathrm{m}}}},
$$

where $k_{\mathrm{d}}$ is the wave number in the dielectric and $\epsilon_{\mathrm{d}}$ and $\epsilon_{\mathrm{m}}$ is the relative permittivity of the dielectric and the metal, respectively [15]. To overcome this momentum mismatch, coupling structures, for example prisms [17,18], or gratings [19] are needed, where in this work grating coupling is performed. For grating coupling the SPP's momentum can be written as

$$
\hbar k_{\mathrm{SPP}}=\hbar k_{\mathrm{d}} \sin (\theta)+\hbar n \frac{2 \pi}{p}
$$

where the surface-parallel component of the photon wave vector, $k_{\mathrm{d}} \sin (\theta)$, is increased by an amount linked to the period $p$ of the grating and the order $n$ of diffraction [20]. Figure 1a depicts a schematic of the reflection of a laser beam at a metal grating. The actual reflection is composed of several orders corresponding to the different values of $n$ with the 0 th, 1 st and -1 st order indicated in the figure. It is important to note that Equation (2) states nothing about the intensity that is given to each mode. This intensity is dependent on the width and the depth of the corresponding grating structure [21]. Those parameters determine the loss rate by radiation of the SPP resonance and must be aligned to accomplish critical coupling [21-23]. Notably, critical coupling can be achieved with various choices of the grating width. Our choice for the duty cycle of 0.43 requires a grating depth of $150 \mathrm{~nm}$ for critical coupling. This in turn results in a convenient range of values for the grating depths (with respect to the fabrication), to study changes of the reflectance as a function of the grating height. Lower or higher duty cycles would require shallower or deeper gratings, respectively, to observe similar behavior of the reflectance and still observe critical coupling. To probe the samples an experimental setup as described in Figure $1 \mathrm{~b}$ is employed (details given in Section 2.3). The surface plot in Figure 1c depicts the simulation results for a silicon grating ( width $=1.6 \mu \mathrm{m}$, period $=2.8 \mu \mathrm{m}$, depth $=50 \mathrm{~nm}$ ) coated with $100 \mathrm{~nm}$ silver. The area of reduced intensity of the reflected light indicates, for which combinations of wavelength and incident angle Equation (2) is fulfilled as the reduced intensity is caused by the excitation of SPPs at the metal surface (0th order). The corresponding literature values for the relative permittivity, for all simulations, are taken from [24].

\subsection{Optical Properties}

In this work we investigate silver as plasmonic material. Figure 2 compares the optical properties of silver $(\mathrm{Ag})$, gold $(\mathrm{Au})$, tungsten $(\mathrm{W})$ and aluminum $(\mathrm{Al})$, namely the real and imaginary part of the relative permittivity $\epsilon_{\mathrm{m}}$. The corresponding literature values for the near- and mid-infrared region are taken from [24]. A high negative real part of the permittivity is an indicator that plasmonic resonances should be observable. The comparison of the mentioned metals shows that silver and aluminum provide the most promising plasmonic properties. On the other hand, the imaginary part of the permittivity states how lossy the materials are. This value increases for increasing wavelength and is similar for gold and silver, with the lowest losses to be expected for tungsten. In this respect aluminum shows the highest losses among the compared materials.

For even better quantification, these optical properties can be used to calculate two typical quality-factors (Q-factors): One for surface plasmon polaritons $\left(Q_{\mathrm{SPP}}=\frac{\operatorname{Re}\left(\epsilon_{\mathrm{m}}\right)^{2}}{\operatorname{Im}\left(\epsilon_{\mathrm{m}}\right)}\right)$ and one for localized surface plasmon resonance $\left(Q_{\mathrm{LSPR}}=\frac{-\operatorname{Re}\left(\epsilon_{\mathrm{m}}\right)}{\operatorname{Im}\left(\epsilon_{\mathrm{m}}\right)}\right)$, both illustrated in Figure $2 \mathrm{~b}$. The corresponding equations are valid for interfaces of metal and air [25]. 
a)

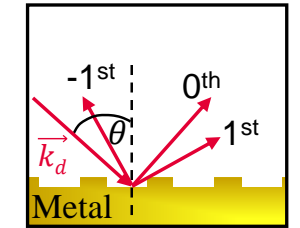

b)

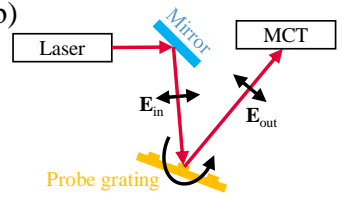

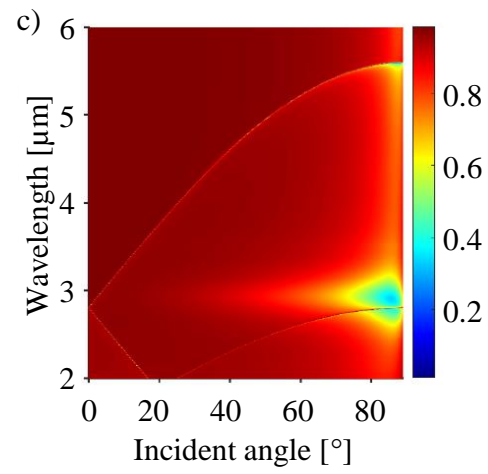

Figure 1. Reflection of radiation at a metal-dielectric interface . (a) Schematic of the reflection of a plane wave at a metal grating (dark yellow). The incident beam is represented by its wave vector $\overrightarrow{k_{d}}$ and incident angle $\theta$. The 0 th, 1 st and -1 st order of reflection are shown. (b) Schematic of the experimental setup. The laser light is guided via mirrors towards the sample grating. The angle of incidence can be adjusted by rotating the sample. The reflected light is collected by a mercury cadmium telluride (MCT) detector. The polarization is chosen perpendicular to the etched grating (TM polarized) and within the plane of incidence (p-polarized). (c) Surface plot depicting the simulated reflectivity of a silver grating as a function of wavelength and incident angle (0th order).
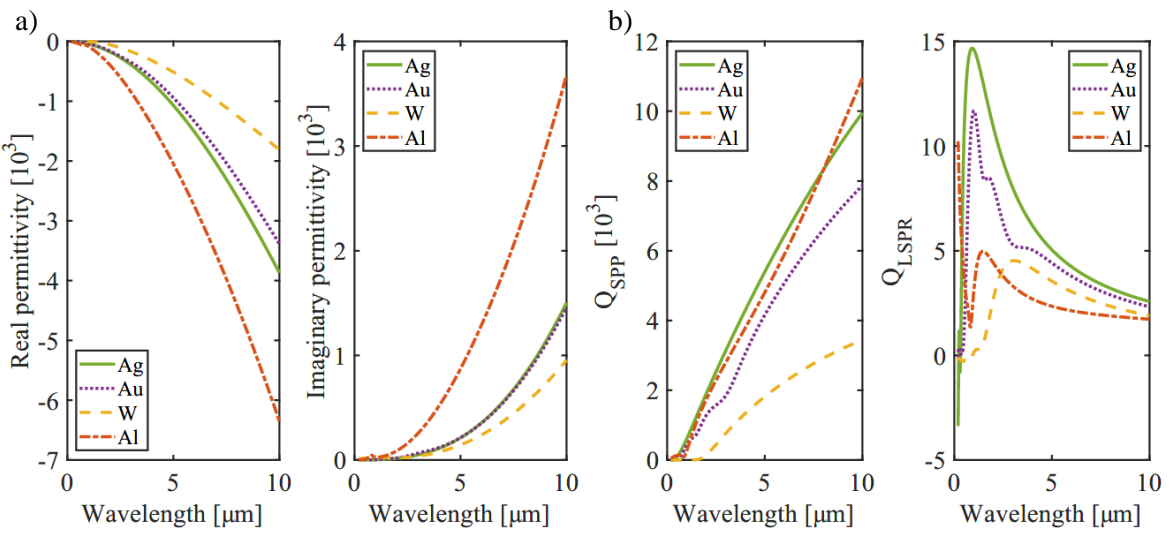

Figure 2. Optical properties of silver $(\mathrm{Ag})$, gold $(\mathrm{Au})$, tungsten $(\mathrm{W})$ and aluminum (Al). (a) Real and imaginary part of the relative permittivity $\epsilon_{\mathrm{m}}$. (b) Localized surface plasmon resonance quality factor $Q_{\mathrm{LSPR}}=\frac{-\operatorname{Re}\left(\epsilon_{\mathrm{m}}\right)}{\operatorname{Im}\left(\epsilon_{\mathrm{m}}\right)}$ and surface plasmon polariton quality factor $Q_{\mathrm{SPP}}=\frac{\operatorname{Re}\left(\epsilon_{\mathrm{m}}\right)^{2}}{\operatorname{Im}\left(\epsilon_{\mathrm{m}}\right)}$ plotted for a wavelength range of $0-10 \mu \mathrm{m}$. Literature values are taken from [24].

Although a high value of $Q_{\text {LSPR }}$ correlates to strong plasmon resonance observable, $Q_{\text {SPP }}$ can be used to calculate the propagation length for SPPs at a certain wavelength. The propagation length $L_{\mathrm{SPP}}$ is given by

$$
L_{\mathrm{SPP}}=\frac{c}{\omega}\left(\frac{\operatorname{Re}\left(\epsilon_{\mathrm{m}}\right)+\epsilon_{\mathrm{d}}}{\operatorname{Re}\left(\epsilon_{\mathrm{m}}\right) \cdot \epsilon_{\mathrm{d}}}\right)^{\frac{3}{2}} \frac{\operatorname{Re}\left(\epsilon_{\mathrm{m}}\right)^{2}}{\operatorname{Im}\left(\epsilon_{\mathrm{m}}\right)},
$$

where $c$ is the speed of light in vacuum and $\omega$ is the angular frequency of the photon [16]. In the regime of $\epsilon_{\mathrm{d}}=1$ and $\left|\epsilon_{\mathrm{m}}\right| \gg \epsilon_{\mathrm{d}}$, the equation can be simplified to

$$
L_{\mathrm{SPP}} \approx \frac{c}{\omega} Q_{\mathrm{SPP}}
$$

As depicted in Figure 2b, silver has the highest values for both Q-factors and is therefore considered to be a promising candidate to observe SPPs. For a wavelength of $4.26 \mu \mathrm{m}$, which is of interest in the context of $\mathrm{CO}_{2}$ sensing, the calculated propagation length is around $990 \mu \mathrm{m}$. Depending on the actual use-case this constitutes a limitation for possible applications. 


\subsection{Experimental Setup and Sample Fabrication}

The experimental setup used to characterize the samples is built according to the schematic given in Figure 1b. The mid-infrared light source is a tunable quantum cascade laser (QCL, MIRcat ${ }^{\mathrm{TM}}$, DRS Daylight Solutions, San Diego, CA, USA), built in a setup for free-beam reflection measurements. Wavelength sweeps are performed in pulsed mode from $3.96 \mu \mathrm{m}$ to $4.35 \mu \mathrm{m}$, covering the whole spectrum of the QCL. The samples are mounted on a manual rotation stage, to control the angle of incidence. The beam position and polarization are controlled by a periscope placed between laser and sample. The polarization is chosen perpendicular to the etched grating (TM polarized) and within the plane of incidence ( $\mathrm{p}$-polarized). The 0th order of reflection is guided to a mercury cadmium telluride (MCT, PVI-4TE-6, VIGO System, Poznan, Poland ) detector. A lock-in amplifier (SR830 DSP, Stanford Research Systems, Sunnyvale, CA, USA) and an oscilloscope (PicoScope, 5000 Series, Pico Technology, Cambridgeshire, UK) are used to perform the detector read out.

The samples are fabricated on eight-inch silicon-substrate wafers in the clean room facilities of Infineon Technologies Austria AG in Villach. A schematic of the layer stack is given in Figure 3a. The silicon substrate is covered by around $2 \mu \mathrm{m}$ of silicon oxide $\left(\mathrm{SiO}_{2}\right)$ for decoupling of the grating structures from the substrate. On top of the $\mathrm{SiO}_{2}, 600 \mathrm{~nm}$ of poly-silicon (poly-Si) is deposited. The actual grating is introduced to the poly-Si by means of lithography and a corresponding dry-etch process. The process duration is adjusted to fabricate gratings of $50 \mathrm{~nm}, 150 \mathrm{~nm}, 225 \mathrm{~nm}$ and $375 \mathrm{~nm}$ depth. After etching and resist removal, the gratings are covered by silver as shown in the schematic of Figure $3 \mathrm{~b}$. The scanning electron microscope (SEM) image in Figure $3 \mathrm{c}$ depicts the etched grating $(50 \mathrm{~nm}$ depth) before metallization. The large grains visible in the grooves is the typical appearance of plasma-etched poly-Si. To have a uniform metal coating, also at the sidewalls of the gratings, the $50 \mathrm{~nm}$ grating is metallized by means of evaporation, whereas for all deeper grating structures sputtering is performed. A SEM image of the cross-section of grating structures with $50 \mathrm{~nm}$ depth, covered with $100 \mathrm{~nm}$ silver (evaporated) is given in Figure $3 \mathrm{~d}$, with a close-up in Figure 3 e.
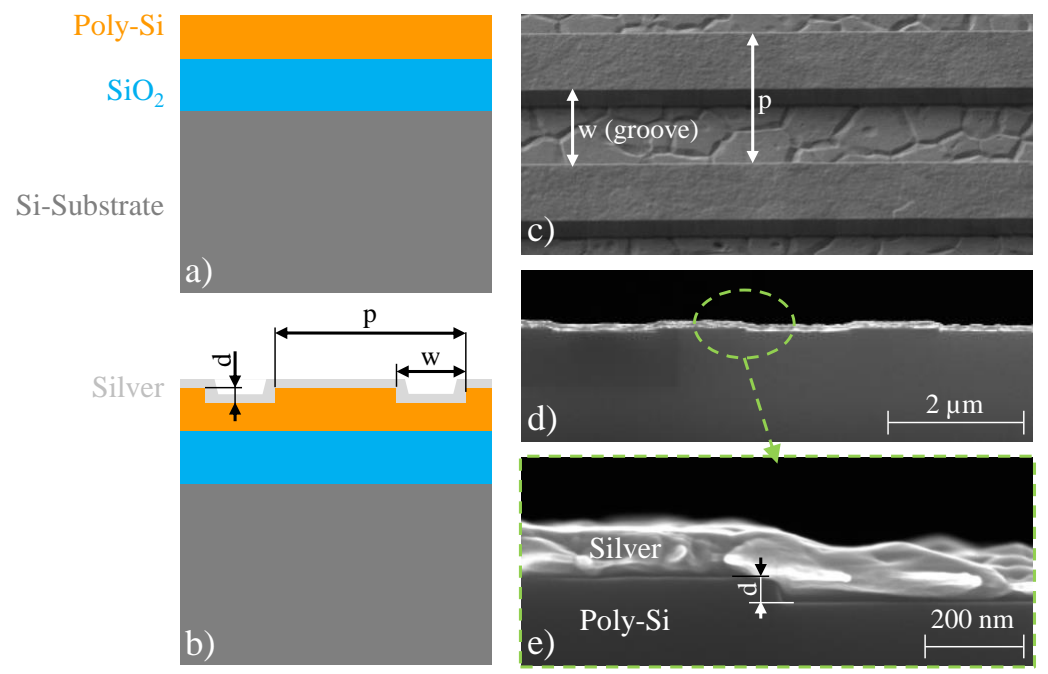

Figure 3. Samples for the layer characterization. (a) Schematic of the layer stack used for fabrication. The eight-inch silicon substrate (dark gray) is covered by around $2 \mu \mathrm{m}$ of silicon oxide $\left(\mathrm{SiO}_{2}\right.$, blue) and $600 \mathrm{~nm}$ of poly-silicon (Poly-Si, orange). (b) The poly-Si is structured by means of lithography and dry etching. The poly-Si grating is covered with silver (light gray). The dimensions drawn are the depth $d$, the width $w$ and the period $p$ of the grating. (c) Scanning electron microscope (SEM) image of etched grating structures ( $50 \mathrm{~nm}$ depth, $p=2.8 \mu \mathrm{m}, w=1.6 \mu \mathrm{m}$ ). The SEM is taken before metallization with a tilt of $45^{\circ}$. (d) SEM cross-section picture of a similar grating structure as shown in (c), uniformly covered by $100 \mathrm{~nm}$ silver (evaporated). (e) Close-up of the region indicated by the green-dashed ellipse in (d). 


\section{Results and Discussion}

With the experimental setup described above, we investigated the wavelength and angular dependence of the reflection on the different samples. The wavelength of the QCL can be adjusted with such an accuracy that no error contribution from wavelength uncertainty is included in the discussion. The incident angle is varied by manually rotating the sample and the error is estimated to be $\pm 1^{\circ}$. The retrieved data are compared to simulations, carried out with the rigorous coupled-wave analysis (RCWA) (DiffractMOD package, Synopsys' RSoft, Mountain View, CA, USA). For all measurements presented in this section the QCL covers the same wavelength range from $3.96 \mu \mathrm{m}$ to $4.35 \mu \mathrm{m}$. In Figures 4, 6 and 7, the panels on the left represent measured values, whereas the right one depicts results retrieved from simulation. For wavelengths beyond $4.25 \mu \mathrm{m}$, the absorption of $\mathrm{CO}_{2}$ present in the ambient air is perceptible in the data. The presented data are connected to the preliminary first experimental results published in [26].

Figure 4 depicts the results of grating structures of $375 \mathrm{~nm}, 225 \mathrm{~nm}$ and $150 \mathrm{~nm}$ depth. The incident angle is varied from $27^{\circ}$ to $32^{\circ}$. According to Equation (2), the resonance wavelength for SPP coupling increases as the incident angle does. This can be seen in the data as the resonance dip shifts by around $220 \mathrm{~nm}$ for an angle change of $5^{\circ}$. The sharp dip in the reflectance spectra corresponds to the SPP resonance, whereas the broader feature at shorter wavelength, results from diffraction into the -1st order. Especially for grating depths $375 \mathrm{~nm}$ and $225 \mathrm{~nm}$ (Figure $4 \mathrm{a}, \mathrm{b}$ ), the intensity of the -1 st order (orange, dashed line in the right panels) of reflection cannot be neglected for wavelengths shorter than the one of SPP resonance. For the grating with $150 \mathrm{~nm}$ depth (Figure $4 \mathrm{c}$ ) the intensity of the -1 st order of reflection becomes much weaker. As a result, the decrease in intensity due to the SPP resonance becomes much more striking. At $27^{\circ}$ of incident angle, the measured intensity of the reflected light has a level of around $80 \%$ at a wavelength of $4 \mu \mathrm{m}$. The intensity drops to close $65 \%$ at $4.020 \mu \mathrm{m}$ wavelength. The center of the feature is located at $4.036 \mu \mathrm{m}$ with a full width at half maximum (FWHM) of $5.5 \mathrm{~nm}$ and a minimum intensity of around $20 \%$. For wavelengths beyond $4.046 \mu \mathrm{m}$ the intensity approaches $100 \%$. The FWHM and center position of the resonance are retrieved from fitting the data with a Fano model $[27,28]$. The same analysis can be performed for the simulated data. For $27^{\circ}$ incidence the simulated data predict the center at $4.077 \mu \mathrm{m}$ with a FWHM of $5.2 \mathrm{~nm}$ and a vanishing intensity.

The weaker attenuation observable in the measured data with respect to the simulation has several reasons: besides roughness of the silver layers and potential worse optical properties of the deposited Ag films, not perfectly polarized laser light can have an influence. All simulations assume perfect transverse magnetic (TM) polarization of the incident light with infinite grating periods. For deeper grating structures, the resonances become wider, which agrees with the theoretical expectation [21].

As a tendency, reducing the grating depth results in narrower SPP resonances. To use this effect to shape the line width of the resonance we perform additional numerical investigation. Figure 5 a depicts the simulated dependence for the 0th order of reflection (specular reflection) on the grating depth. For the target wavelength range of $3.96 \mu \mathrm{m}$ to $4.35 \mu \mathrm{m}$, a grating depth of $50 \mathrm{~nm}$ is predicted to reveal resonances in the range of $1.4 \mathrm{~nm}$ FWHM and a signal attenuation of around 30\%, for an angle of incidence of $28^{\circ}$ (see Figure $5 b$ left panel). Figure $5 b$ represents a comparison of the simulated (left panel) and measured (right panel) SPP resonance feature in reflection for the grating of $50 \mathrm{~nm}$ depth at an incident angle of $28^{\circ}$. 

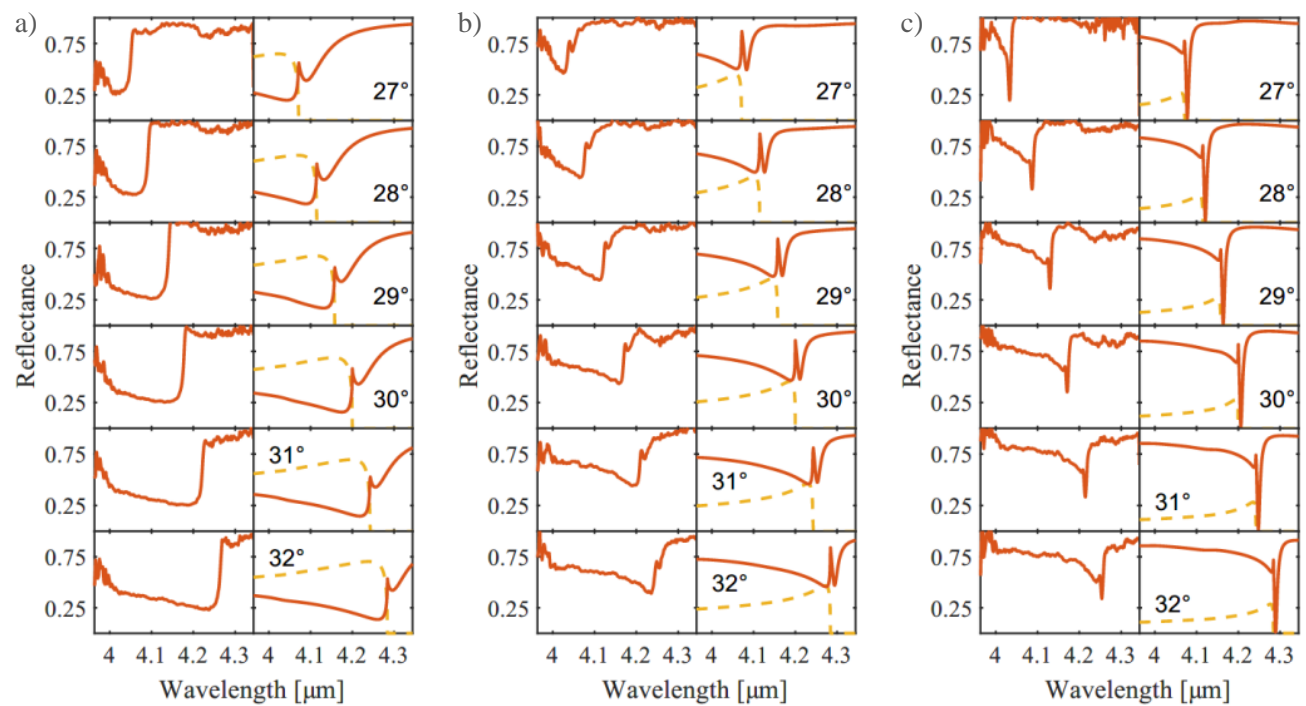

Figure 4. Measurement results and simulations for different grating depths. Each left panel represents the measured intensity of the 0th order of reflection (red). The simulated data are given in the right panels, consisting of the corresponding 0th (red) and the -1st (orange, dashed) order. The tested samples are silver-coated (100 nm, sputtered) grating structures of (a) $375 \mathrm{~nm}$, (b) $225 \mathrm{~nm}$ and (c) $150 \mathrm{~nm}$ depth. The incident angle of the laser beam with respect to the sample surface is varied from $27^{\circ}$ to $32^{\circ}$. The wavelength is tuned from $3.96 \mu \mathrm{m}$ to $4.35 \mu \mathrm{m}$.

a)

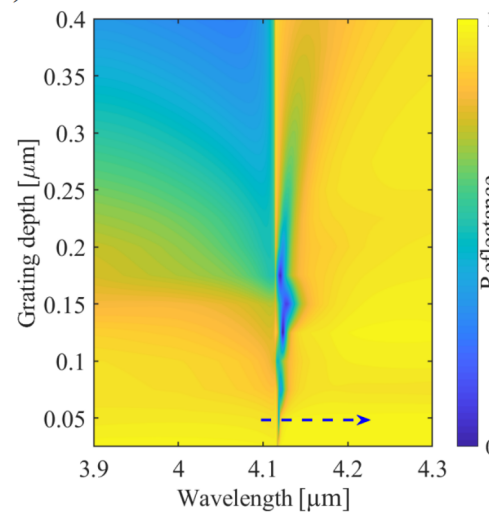

b)

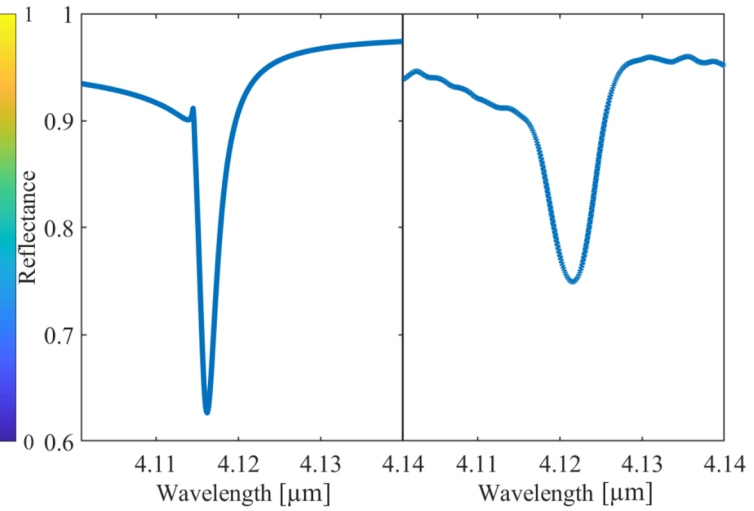

Figure 5. Optimization of grating depth to retrieve ultra-narrow SPP resonances for potential sensing applications. (a) Simulated dependence of 0th order reflectance (specular reflectance) at $28^{\circ}$ on the grating depth. (b) Simulated (left panel) and measured (right panel) specular reflectance at $28^{\circ}$ of the grating of $50 \mathrm{~nm}$ depth $(p=2.8 \mu \mathrm{m}, w=1.6 \mu \mathrm{m})$.

Therefore, we further investigate a shallower silver grating of $50 \mathrm{~nm}$ depth. In Figure 6, a similar comparison of measured values and simulation is depicted. The measurement covers an angle variation in the range of $26^{\circ}$ to $33^{\circ}$. The resonance dip shifts by around $280 \mathrm{~nm}$ for an angle change of $7^{\circ}$. The SPP resonance at $28^{\circ}$ of incident angle reduces the intensity of the reflected light from close $96 \%$ (off-resonant) to a level of around $75 \%$ which translates to an attenuation of $21 \%$. The feature has a FWHM of $2.9 \mathrm{~nm}$ and is centered at a wavelength of $4.122 \mu \mathrm{m}$. The simulation reveals a FWHM of $1.4 \mathrm{~nm}$ with the resonance center at $4.116 \mu \mathrm{m}$ and a minimum intensity of $63 \%$. 


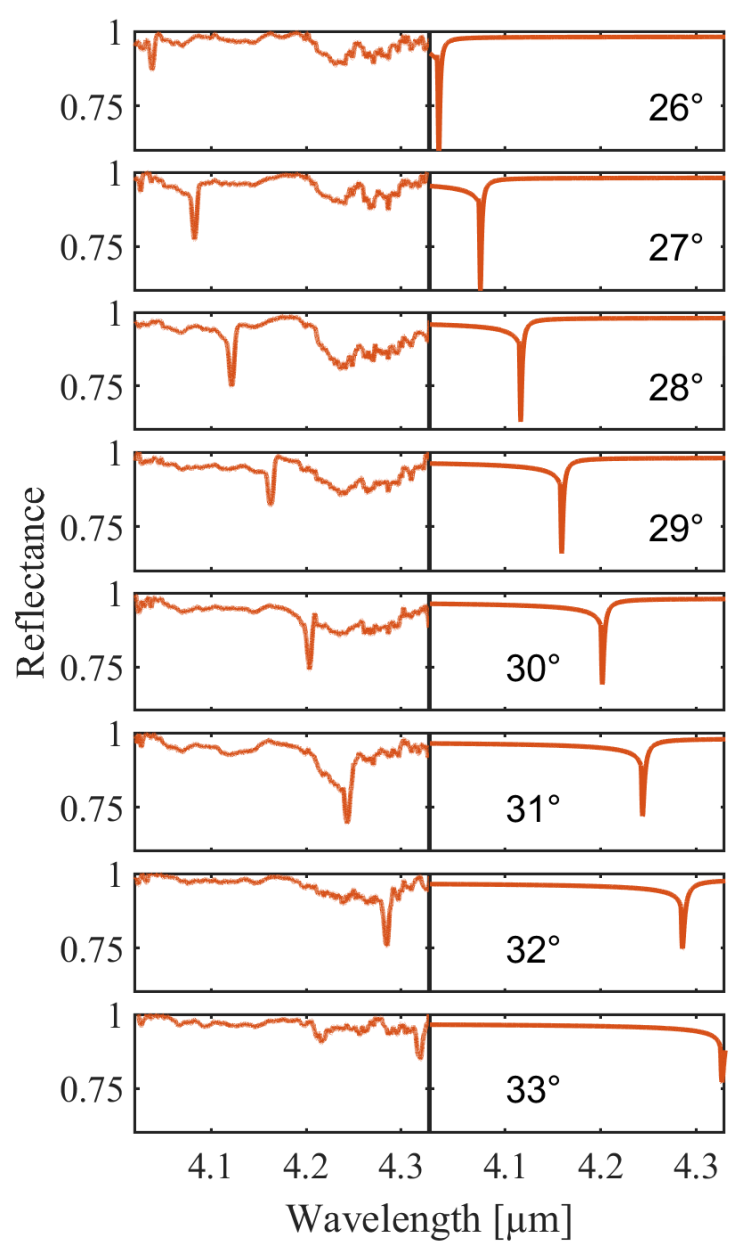

Figure 6. Comparison of measurement results (left panel) and simulated data (right panel) for a silver-coated (100 nm, evaporated) grating of $50 \mathrm{~nm}$ depth. The incident angle of the laser beam with respect to the sample surface is varied from $26^{\circ}$ to $33^{\circ}$ and the wavelength is tuned from $3.96 \mu \mathrm{m}$ to $4.35 \mu \mathrm{m}$. The panels represent a reflectance of $60-100 \%$.

The surface plots in Figure 7 illustrate the specular reflectance of the same grating sample of $50 \mathrm{~nm}$ depth coated with silver in a wider range of incident angles and wavelengths. The wavelength regime for this measurement reaches from $2 \mu \mathrm{m}$ to $6 \mu \mathrm{m}$ and the angle of incidence is varied from $25^{\circ}$ to $80^{\circ}$ with a step-size of $5^{\circ}$. The measured and simulated values are given in Figure $7 \mathrm{a}, \mathrm{b}$, respectively. As already mentioned and explained for Figure 1c, the reduced reflection is caused by the excitation of SPPs at the interface. The lines of reduced intensity within these plots indicate, for which combinations of wavelength and incident angle Equation (2) is fulfilled. The measurement is done with a FTIR ellipsometer (IR-VASE, J.A. Woollam Co., Lincoln, NE, USA). Even if the absolute values of reflected intensity are different, the measured values (left panel) are in good agreement with the simulated ones (right panel). Since the excitation beam in the FTIR ellipsometer is not a coherent light source, the signal to noise ratio is lower (shallower resonance with higher noise floor) compared to the reflectance measurement using the QCL. The main source of noise within the experimental setup is the unwanted absorption of the light by $\mathrm{CO}_{2}$ and water vapor present in the laboratory's atmosphere. The bright, almost angle independent feature at $3 \mu \mathrm{m}$ is attributed to the higher order of the localized surface plasmon mode. 

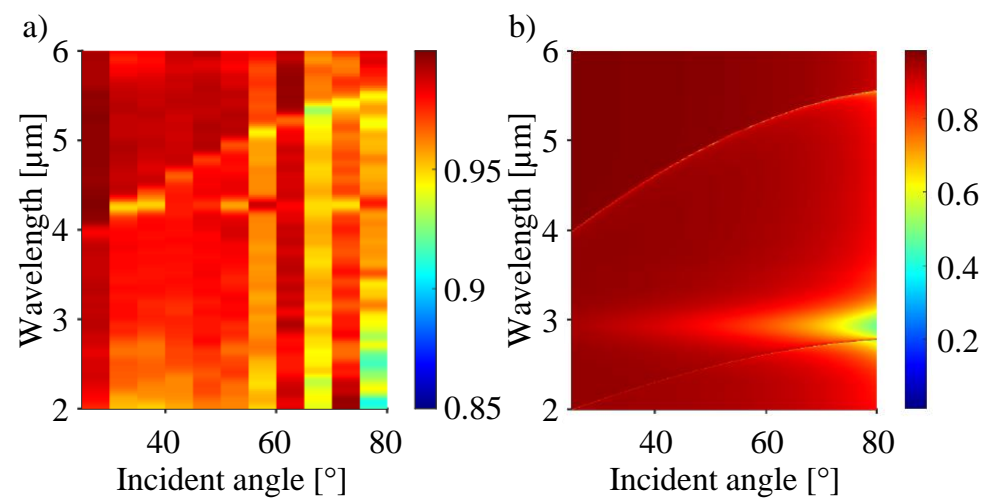

Figure 7. Surface plot depicting the reflectivity of a grating with $50 \mathrm{~nm}$ depth, coated with $100 \mathrm{~nm}$ silver (evaporated). Plotted is the wavelength and incident angle for the 0th order of reflection. The areas of reduced intensity are due to the excitation of SPPs at the metal's surface. $(\mathbf{a}, \mathbf{b})$ Representation of measured values and a corresponding simulation, respectively.

\section{Potential Applications for Sensing}

The presented measurements and the material properties of plasmonic layers in general allow various sensing applications based on plasmonics and SPPs. Previous works suggest different use-cases, for example thin-film measurements or refractive index sensing $[4,21,29]$. As an example, we numerically investigate the possibility to use these structures as a platform for refractive index sensing. Changes in the refractive index in the cladding introduce spectral shifts in the SPP resonance. For high sensitivity, ultranarrow SPP resonances are needed, which can be retrieved by optimizing the grating parameters. As suggested by the simulation presented in the beginning, we indeed see such ultra-narrow SPP resonances for the grating with $50 \mathrm{~nm}$ depth at an incident angle of $28^{\circ}$ (see Figure $5 a, b$ ). Further numerical investigations concerning refractive index sensing with respect to our grating samples (50 nm depth) are depicted in Figure 8a,b. Changes of the refractive index of the dielectric between 1 and 1.010 refractive index units (RIUs) can be resolved with an average spectral shift of $4122 \frac{\mathrm{nm}}{\mathrm{RIU}}$. In the field of refractive index sensing, tuning the plasmon line shape is used to narrow down the line widths. This in turn results in a higher figure of merit $\left(F O M=\frac{\frac{d \lambda}{d n}}{F W H M}\right)$ and therefore lowers the detection limit [30]. In our case the FOM is around $1421 \mathrm{RIU}^{-1}[12,30,31]$. However, comparing the retrieved results for the grating with $150 \mathrm{~nm}$ and $50 \mathrm{~nm}$ depth, it can be seen that there is an optimum depth at a given period, for which the amplitude of the resonance reaches its maximum. Even though the grating with $50 \mathrm{~nm}$ depth reveals narrower resonances, the grating with $150 \mathrm{~nm}$ depth would enable a higher signal to noise ratio achievable for a sensor application. The mentioned range from 1 to 1.010 RIU was used only to determine the differential sensitivity of the proposed structure and it does not correspond to a meaningful change in the ambient atmosphere. 

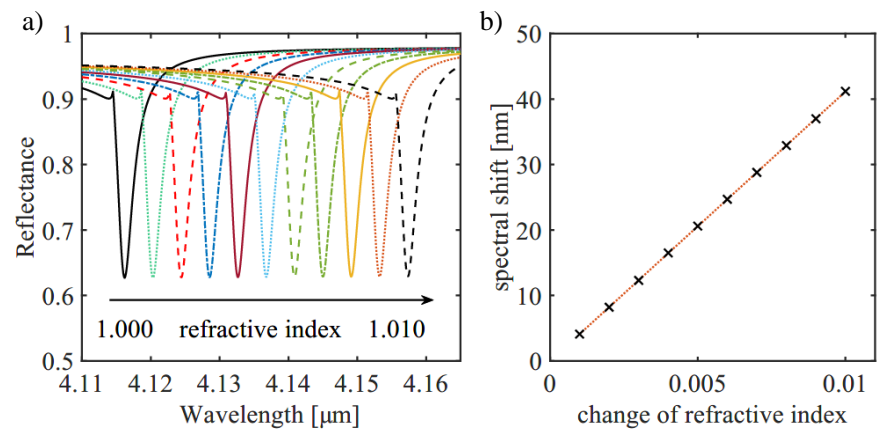

Figure 8. Numerical investigation of refractive index sensing based on the shallow grating structures of $50 \mathrm{~nm}$ depths. (a) Simulation of the spectral shift of the SPP resonance due to slight changes of the refractive index $n$ of the dielectric. The refractive index is changed from 1.000 refractive index unit (RIU) to 1.010 RIU. (b) From the simulation in (a), an average spectral shift of $4122 \frac{\mathrm{nm}}{\mathrm{RIU}}$ can be determined.

\section{Conclusions}

In summary, we have successfully demonstrated the excitation of ultra-narrow SPP resonances from plasmonic, metalized, thin-film gratings in the MIR region for, e.g., sensing applications. Motivated by simulations on optical properties of different metals, we identified silver as a proper material of choice, since the optical properties of this metal are promising with respect to the excitation of SPPs in the MIR region. The fabricated samples covered different grating depths, $50 \mathrm{~nm}, 150 \mathrm{~nm}, 225 \mathrm{~nm}$ and $375 \mathrm{~nm}$, which allows active shaping of the line width of the SPP resonance. We shortly discussed the theoretical background and corresponding numerical calculations. The experimental part covered the wavelength range from $3.96 \mu \mathrm{m}$ to $4.35 \mu \mathrm{m}$ in the mid-infrared part of the spectrum. The retrieved experimental data are in good agreement with the simulations carried out. This is found for the layer properties in general, the dependence of the resonance on the angle of incidence as well as on the wavelength. The expected SPP resonances can be observed as a reduced intensity of the specular reflectance. In particular, the grating with a depth of $50 \mathrm{~nm}$ revealed SPP resonances with a FWHM of $2.9 \mathrm{~nm}$ and a signal attenuation of $21 \%$. SPP resonances of this kind, with an ultra-narrow line width and a pronounced signal attenuation, are needed to be of use for practical sensing applications. In this context, we further investigated the feasibility to use the suggested grating structures as a platform for refractive index sensing. Indeed, the calculated sensitivity of a sensor based on the grating with a depth of $50 \mathrm{~nm}$ is found as high as $4122 \frac{\mathrm{nm}}{\mathrm{RIU}}\left(\mathrm{FOM}=1421 \mathrm{RIU}^{-1}\right)$. In addition to the promising results from the experiments and simulations presented, which show that the examined samples represent an interesting platform for future sensor applications, we would like to state that the entire fabrication took place in the industrial clean room facilities of Infineon Technologies Austria AG. Therefore, this work also proofs that plasmonic sensor technologies are compatible with the standard processes and mass production of the state-of-the-art semiconductor industry.

Author Contributions: Conceptualization, G.S., T.G., R.J., G.P., A.T. and C.C.; methodology, G.S., J.S., C.F., C.C. and T.D.D.; software, T.D.D., F.D., R.J., G.P. and P.S.; resources, E.A., T.G. and B.J.; data curation, G.S., J.S. and T.D.D.; writing-original draft preparation, G.S. and J.S.; writing-review and editing, All authors; supervision, B.J. and A.T.; project administration, E.A., T.G., B.J., G.S. and A.T.; funding acquisition, E.A., T.G., A.T. and B.J. All authors have read and agreed to the published version of the manuscript.

Funding: This work was performed within the PICASSO-project funded by the Bundesministerium für Klimaschutz, Umwelt, Energie, Mobilität, Innovation und Technologie (BMK) in the framework of the program "Produktion der Zukunft" (Prj. Nr. 871417).

Institutional Review Board Statement: Not applicable. 
Informed Consent Statement: Not applicable.

Data Availability Statement: Not applicable.

Acknowledgments: The authors want to thank all the involved process experts working in the cleanroom facilities of Infineon Technologies Austria AG, Villach, for their contributions to discussions and support during fabrication-especially our colleagues from layout, lithography, etching and oven processes. Jasmin Spettel wants to thank Peter Hadley (Graz University of Technology) for his kind advice.

Conflicts of Interest: The authors declare no conflict of interest.

\section{References}

1. Tong, L.; Wei, H.; Zhang, S.; Xu, H. Recent Advances in Plasmonic Sensors. Sensors 2014, 14, 7959-7973. [CrossRef] [PubMed]

2. Ibrahim, J.; Al Masri, M.; Verrier, I.; Kampfe, T.; Veillas, C.; Celle, F.; Cioulachtjian, S.; Lefèvre, F.; Jourlin, Y. Surface Plasmon Resonance Based Temperature Sensors in Liquid Environment. Sensors 2019, 19, 3354. [CrossRef]

3. Liu, Y.; Ma, Y. One-Dimensional Plasmonic Sensors. Front. Phys. 2020, 8, 312. [CrossRef]

4. Liu, N.; Mesch, M.; Weiss, T.; Hentschel, M.; Giessen, H. Infrared Perfect Absorber and Its Application As Plasmonic Sensor. Nano Lett. 2010, 10, 2342-2348.

5. Martínez, J.; Ródenas, A.; Aguiló, M.; Fernandez, T.; Solis, J.; Díaz, F. Mid-infrared surface plasmon polariton chemical sensing on fiber-coupled ITO coated glass. Opt. Lett. 2016, 41, 2493-2496. [CrossRef]

6. Wan, B.F.; Zhou, Z.W.; Xu, Y.; Zhang, H.F. A Theoretical Proposal for a Refractive Index and Angle Sensor Based on OneDimensional Photonic Crystals. IEEE Sens. J. 2021, 21, 331-338. [CrossRef]

7. Wan, B.F.; Xu, Y.; Zhou, Z.W.; Zhang, D.; Zhang, H.F. Theoretical Investigation of a Sensor Based on One-Dimensional Photonic Crystals to Measure Four Physical Quantities. IEEE Sens. J. 2021, 21, 2846-2853. [CrossRef]

8. Ranacher, C.; Consani, C.; Tortschanoff, A.; Jannesari, R.; Bergmeister, M.; Grille, T.; Jakoby, B. Mid-infrared absorption gas sensing using a silicon strip waveguide. Sens. Actuators Phys. 2018, 277, 117-123. [CrossRef]

9. Miyazaki, H.T.; Ikeda, K.; Kasaya, T.; Yamamoto, K.; Inoue, Y.; Fujimura, K.; Kanakugi, T.; Okada, M.; Hatade, K.; Kitagawa, S. Thermal emission of two-color polarized infrared waves from integrated plasmon cavities. Appl. Phys. Lett. 2008, $92,141114$. [CrossRef]

10. Liu, X.; Tyler, T.; Starr, T.; Starr, A.F.; Jokerst, N.M.; Padilla, W.J. Taming the Blackbody with Infrared Metamaterials as Selective Thermal Emitters. Phys. Rev. Lett. 2011, 107, 045901. [CrossRef]

11. Ranacher, C.; Consani, C.; Tortschanoff, A.; Rauter, L.; Holzmann, D.; Fleury, C.; Stocker, G.; Fant, A.; Schaunig, H.; Irsigler, P.; et al. A CMOS Compatible Pyroelectric Mid-Infrared Detector Based on Aluminium Nitride. Sensors 2019, 19, 2513. [CrossRef]

12. Cheng, F.; Yang, X.; Gao, J. Enhancing intensity and refractive index sensing capability with infrared plasmonic perfect absorbers. Opt. Lett. 2014, 39, 3185-3188. [CrossRef]

13. Cerjan, B.; Yang, X.; Nordlander, P.; Halas, N.J. Asymmetric Aluminum Antennas for Self-Calibrating Surface-Enhanced Infrared Absorption Spectroscopy. ACS Photonics 2016, 3, 356-360. [CrossRef]

14. Stocker, G.; Spettel, J.; Dao, T.D.; Tortschanoff, A.; Jannesari, R.; Pühringer, G.; Saeidi, P.; Dubois, F.; Fleury, C.; Consani, C.; et al. Plasmonic Silver Grating for Mid-Infrared Sensing. In Proceedings of the 21st International Conference on Solid-State Sensors, Actuators and Microsystems (Transducers), Orlando, FL, USA, 20-24 June 2021; pp. 1072-1075. [CrossRef]

15. Maier, S.A. Plasmonics: Fundamentals And Applications; Springer: New York, NY, USA, 2007; [CrossRef]

16. Barnes, W.L.; Dereux, A.; Ebbesen, T.W. Surface plasmon subwavelength optics. Nature 2003, 424, 824- 830. [CrossRef] [PubMed]

17. Kretschmann, E.; Raether, H. Radiative Decay of Non Radiative Surface Plasmons Excited by Light. Z. Naturfaroschung 1968, 23, 2135-2136. [CrossRef]

18. Otto, A. Excitation of nonradiative surface plasma waves in silver by the method of frustrated total reflection. Z. Physik 1968, 216, 398-410. [CrossRef]

19. Zhang, J.; Zhang, L.; Xu, W. Surface plasmon polaritons: physics and applications. J. Phys. Appl. Phys. 2012, 45, 113001. [CrossRef]

20. Sambles, J.R.; Bradbery, G.W.; Yang, F. Optical excitation of surface plasmons: An introduction. Contemp. Phys. 1991, 32, 173-183. [CrossRef]

21. Meng, L.; Zhao, D.; Ruan, Z.; Li, Q.; Yang, Y.; Qiu, M. Optimized grating as an ultra-narrow band absorber or plasmonic sensor. Opt. Lett. 2014, 39, 1137-1140. [CrossRef] [PubMed]

22. Fan, S.; Suh, W.; Joannopoulos, J.D. Temporal coupled-mode theory for the Fano resonance in optical resonators. J. Opt. Soc. Am. A 2003, 20, 569-572. [CrossRef] [PubMed]

23. Yoon, J.; Seol, K.H.; Song, S.H.; Magnusson, R. Critical coupling in dissipative surface-plasmon resonators with multiple ports. Opt. Express 2010, 18, 25702-25711. [CrossRef]

24. Rakić, A.D.; Djurišić, A.B.; Elazar, J.M.; Majewski, M.L. Optical properties of metallic films for vertical-cavity optoelectronic devices. Appl. Opt. 1998, 37, 5271-5283. [CrossRef] 
25. West, P.; Ishii, S.; Naik, G.; Emani, N.; Shalaev, V.; Boltasseva, A. Searching for better plasmonic materials. Laser Photonics Rev. 2010, 4, 795-808. [CrossRef]

26. Spettel, J.; Stocker, G.; Dao, T.D.; Jannesari, R.; Tortschanoff, A.; Saeidi, P.; Pühringer, G.; Dubois, F.; Fleury, C.; Consani, C.; et al. Aluminium, gold-tin and titanium-tungsten alloys for mid-infrared plasmonic gratings. Opt. Mater. Express 2021, 11, $1058-1069$. [CrossRef]

27. Pusep, Y.; Rodrigues, A.; Borrero-Gon, L.; Acquaroli, L.; Urteaga, R.; Arce, R.; Koropecki, R.; Tirado, M.; Comedi, D. Fano resonance in heavily doped porous silicon. J. Raman Spectrosc. 2011, 42, 1405. [CrossRef]

28. Racu, A.M.; Menzel, D.; Schoenes, J.; Doll, K. Crystallographic disorder and electron-phonon coupling in $\mathrm{Fe}_{1-\mathrm{x}} \mathrm{Co}_{\mathrm{x}} \mathrm{Si}$ single crystals: Raman spectroscopy study. Phys. Rev. B 2007, 76, 115103. [CrossRef]

29. Yue, W.; Wang, Z.; Yang, Y.; Han, J.; Li, J.; Guo, Z.; Tan, H.; Zhang, X.X. High Performance Infrared Plasmonic Metamaterial Absorbers and Their Applications to Thin-film Sensing. Plasmonics 2016, 11, 1557-1563. [CrossRef]

30. Lodewijks, K.; Ryken, J.; Van Roy, W.; Borghs, G.; Lagae, L.; Van Dorpe, P. Tuning the Fano Resonance Between Localized and Propagating Surface Plasmon Resonances for Refractive Index Sensing Applications. Plasmonics 2013, 8, 1379-1385. [CrossRef]

31. Wen, Y.; Sun, Y.; Deng, C.; Huang, L.; Hu, G.; Yun, B.; Zhang, R.; Cui, Y. High sensitivity and FOM refractive index sensing based on Fano resonance in all-grating racetrack resonators. Opt. Commun. 2019, 446, 141-146. [CrossRef] 